\title{
Low-Complexity Iterative Receiver for Space-Time Coded Signals over Frequency Selective Channels
}

\author{
Noura Sellami \\ France Télécom R\&D, 38-40 rue du Général Leclerc, 92794 Issy les Moulineaux, France \\ Équipe de Traitement des Images et du Signal, ENSEA-Université de Cergy Pontoise, \\ 6 avenue du Ponceau, 95014 Cergy-Pontoise, France \\ Email:noura.sellami@rd.francetelecom.com \\ Inbar Fijalkow \\ Équipe de Traitement des Images et du Signal, ENSEA-Université de Cergy Pontoise, \\ 6 avenue du Ponceau, 95014 Cergy-Pontoise, France \\ Email: fijalkow@ensea.fr \\ Mohamed Siala \\ Sup'Com, Route de Raoued Km 3.5, 2083 Ariana, Tunisia \\ Email: mohamed.siala@supcom.rnu.tn
}

Received 31 May 2001 and in revised form 18 March 2002

\begin{abstract}
We propose a low-complexity turbo-detector scheme for frequency selective multiple-input multiple-output channels. The detection part of the receiver is based on a List-type MAP equalizer which is a state-reduction algorithm of the MAP algorithm using per-survivor technique. This alternative achieves a good tradeoff between performance and complexity provided a small amount of the channel is neglected. In order to induce the good performance of this equalizer, we propose to use a whitened matched filter (WMF) which leads to a white-noise "minimum phase" channel model. Simulation results show that the use of the WMF yields significant improvement, particularly over severe channels. Thanks to the iterative turbo processing (detection and decoding are iterated several times), the performance loss due to the use of the suboptimum List-type equalizer is recovered.
\end{abstract}

Keywords and phrases: space-time coded MIMO channel equalization, per-survivor processing, multidimensional whitened matched filter, turbo detection.

\section{INTRODUCTION}

The growing demand for new services at high data rates indicates the need for new techniques to increase channel capacity. Foschini and Gans [1] have demonstrated the enormous capacity potential gain of wireless communication systems with antenna arrays at both transmitter and receiver. In order to achieve the promised high data rates over frequency selective multiple-input multiple-output (MIMO) channels, an equalizer has to be applied to reduce the channel time dispersion due to multipath propagation at high data rates.

Several solutions have been proposed among them linear and decision-feedback structures with a zero forcing or minimum mean square error optimization [2]. These equalizers have a low-complexity but suffer from noise enhancement and error propagation. In terms of performance, it is better to use a maximum a posteriori (MAP) [3] or Viterbi equalizer. However, the complexity of these algorithms is proportional to the number of states of the trellis which grows exponentially with the product of the channel memory and the number of transmit antennas [4]. When the channel memory becomes large and high-order constellations are used, the algorithm becomes impractical. Therefore, a reduced complexity approach is needed.

In this paper, we consider a List-type MAP equalizer [5] which realizes a good tradeoff between performance and complexity. The trellis has a reduced number of states taking into account a reduced number of taps of the channel. The remaining intersymbol and cochannel interference is cancelled by an internal per-survivor processing with a list of $S$ survivors $[6,7,8]$. The choice of the receiver filter can affect dramatically the performance of this suboptimal equalizer. To induce the performance of this equalizer, it is desirable to use a receiver filter which concentrates the energy on the first taps. In the scalar case, this is easily achieved using a minimum phase factorization of the channel. Here, we extend this approach to the MIMO case. Therefore, we 


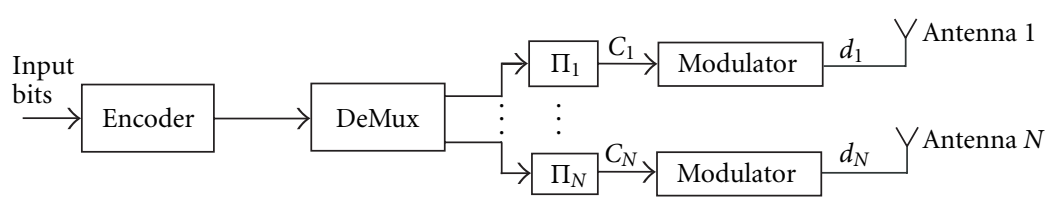

FIgURE 1: Transmitter structure.

propose to use a whitened matched filter (WMF) which makes the channel "minimum phase" and keeps the noise white $[9,10,11]$. Simulation results show that the use of the WMF yields significant improvement particularly over severe channels.

We propose here to enhance the performance by using a channel encoder. As shown in Figure 1, we consider a MIMO system transmitting parallel data coded streams and demultiplexed simultaneously. The structure of this transmitter, referred to as bit-interleaved coded modulation (BICM), was initially proposed for single-antenna systems $[12,13]$. A single code is cyclically connected to $N$ transmitters, each including a bit interleaver $\Pi_{i}$ followed by a modulator and an antenna. The code and the frequency selective channel separated by the interleavers constitute a serially concatenated scheme to which we can apply an iterative receiver composed of a soft-input/soft-output decoder and an equalizer following the idea of turbo-codes [14]. The basic idea behind iterative processing is to exchange extrinsic information among the receiver modules in order to achieve successively refined performance. Turbo processing for MIMO coded data enhances all the more the need for a low-complexity equalizer. In $[15,16]$, an equalizer based on filters has been proposed. However, the gain in complexity induces a loss in performance. In [17], Bauch and Al-Dhahir used a set of shortening filters [18] as prefilters to turbo equalization in order to reduce the number of states of the MAP equalizer. The disadvantage of this method is that the noise becomes colored at the outputs of the shortened channel.

In this paper, we consider a turbo detector composed of a List-type MAP equalizer and a MAP decoder. Thanks to the WMF, the noise at the input of the turbo detector is white. This permits to have a good performance without enhancing the complexity of the equalizer. In fact, in the case of a colored noise, the noise autocorrelation matrix has to be taken into account by the equalizer in order to achieve good performance. Unfortunately, the complexity of the equalizer is then considerably increased. If, for simplicity, the colored noise is assumed to be white, the soft outputs of the equalizer are degraded, particularly on the first turbo iteration. This can affect significantly the performance of the iterative receiver since we know the importance of the equalization at the first iteration for the turbo processing.

The paper is organized as follows. In Section 2, we describe the system model. In Section 3, we present the whitened matched filter principle and give simulation results for the List-type MAP equalizer. In Section 4, we describe the proposed turbo detector and the corresponding simulation results.

Throughout, scalars and matrices are lower and upper case, respectively, and vectors are underlined lower case; $(\cdot)^{T},(\cdot)^{\dagger}$, and $(\cdot)^{-1}$ denote, respectively, transposition, transconjugation, and inversion. Moreover, $\operatorname{Trace}(A)$ denotes the trace of matrix $A$ and $E(\cdot)$ denotes the expected value operator. Finally, $\lfloor x\rfloor$ denotes the highest integer not bigger than $x$.

\section{SYSTEM MODEL}

\subsection{General framework}

We consider a frequency selective fading MIMO channel with $N$ transmitting antennas and $M$ receiving antennas. The channel between each transmit antenna and each receive antenna is modeled by a Rayleigh fading with a memory of $L$ symbols.

As shown in Figure 1, the input information bit sequence is first encoded with a rate $K$ convolutional encoder. The output of the encoder is demultiplexed into $N$ streams that are interleaved by different interleavers $\Pi_{i}$, mapped to PSK/QAM symbols and transmitted simultaneously by the $N$ transmitting antennas. The same modulation constellation with size $Q$ is used for each stream. Thus, $\log _{2}(Q)$ coded bits are mapped into one $Q$-ary symbol. This scheme was first proposed in $[12,13]$ for single-antenna and is known as bit-interleaved coded modulation (BICM). We assume that transmissions are organized into bursts of $T$ symbols. For the sake of simplicity, the channels are supposed to be invariant during a burst and to change independently from burst to burst.

The received baseband signal sampled at the symbol rate at antenna $j$ at time $k$ is a linear combination of the $N$ transmitted signals perturbed with noise

$$
r_{j}(k)=\sum_{i=1}^{N} \sum_{l=0}^{L-1} h_{i, j}(l) d_{i}(k-l)+n_{j}(k) .
$$

In this expression, $n_{j}(k)$ are modeled as independent samples of a zero mean white complex Gaussian noise with variance $\sigma^{2}=N_{0}$ and $h_{i, j}(l)$ is the lth tap gain from transmit antenna $i$ to receive antenna $j$. The tap gains $h_{i, j}(l)$ are modeled as independent complex Gaussian random variables with zero mean and variance $\sigma_{h}^{2}(l)$. We assume that $\sum_{l=0}^{L-1} \sigma_{h}^{2}(l)=1$. Let $\underline{d}(k)=\left(d_{1}(k), \ldots, d_{N}(k)\right)^{T}$ be the $N$-long vector of modulated symbols transmitted from the $N$ transmitting antennas at time $k$ and $\underline{n}(k)=\left(n_{1}(k), \ldots, n_{M}(k)\right)^{T}$ be the $M$-long noise vector at the receiving antennas.

The output of the channel is the $M$-long vector $\underline{r}(k)=$ $\left(r_{1}(k), \ldots, r_{M}(k)\right)^{T}$ with $Z$-transform:

$$
\underline{r}(z)=H(z) \underline{d}(z)+\underline{n}(z),
$$

where $H(z)=\sum_{l=0}^{L-1} H(l) z^{-l}$ and $(H(l))_{j, i}=h_{i, j}(l)$. 
The problem we address is then to recover the information bits from the noisy observation.

\subsection{Simulation framework}

In our simulations, we concentrate on a MIMO system with 2 transmit antennas and 2 receive antennas. We use a frequency-selective fading channel with memory $L=5$. The channel is considered to be time invariant during the transmission of a burst of 512 information bits and changes independently from burst to burst. We assume that the channel is perfectly known at the receiver. The rate $K=1 / 2$ convolutional code has 4 states and generator polynomials $(7,5)$. The modulation used is BPSK. We plot the bit error rate (BER) with respect to averaged $E_{b} / N_{0}$ per receive antenna.

\section{EQUALIZATION}

In the case of a single-input single-output (SISO) frequency selective channel, a whitened matched filter (WMF) is used to transform the received signal into a sequence with minimum phase channel response and additive white noise. This procedure is a first step in the implementation of some equalizers including decision-feedback detectors and delayed decision-feedback sequence estimators (DDFSE) [6]. This is achieved by factoring the channel spectrum into a product of a minimum phase filter and its time inverse. In this paper, we propose to use a List-type equalizer which realizes a good tradeoff between performance and complexity. Since this type of algorithm considers a reduced number of states corresponding to a reduced number of taps of the channel $[6,7,8]$, it is desirable to design a multidimensional WMF and to use it as a prefilter for the equalizer, analogously to the scalar case. In the following, we will present a solution based on prediction theory results.

\subsection{Whitened matched filter}

As in the SISO case, the first step is to feed the received signal $\underline{r}(z)$ to the matched filter $H^{\dagger}\left(z^{-1}\right)$. The output signal is then,

$$
H^{\dagger}\left(z^{-1}\right) \underline{r}(z)=\underline{x}(z)=S(z) \underline{d}(z)+\underline{b}(z),
$$

where $S(z)=H^{\dagger}\left(z^{-1}\right) H(z)$ and $\underline{b}(z)=H^{\dagger}\left(z^{-1}\right) n(z)$ is a colored noise with spectrum $N_{0} S(z)$.

For the multidimensional case, some results have been derived using the linear prediction theory for vector widesense stationary (WSS) processes $[9,10,11]$. An interesting result is stated below.

Theorem 1 (multidimensional spectral factorization [11]). Given an $N$-dimensional WSS process $\underline{v}(z)$ with spectrum $S_{v}(z)$, there exists a factorization

$$
S_{v}(z)=B_{-}^{\dagger}\left(z^{-1}\right) B_{-}(z)
$$

such that $B_{-}(z)$ is causal and stable and has a causal inverse. The filter $B_{-}^{-1}(z)$ is stable if $S_{v}(z)$ is nonsingular on the unit circle. This factorization is called the minimum phase factorization of $S_{v}(z)$.
According to this theorem, there exists an $(N \times N)$ causal and stable matrix filter $B_{-}(z)$ with causal and stable inverse which verifies $B_{-}^{\dagger}\left(z^{-1}\right) B_{-}(z)=H^{\dagger}\left(z^{-1}\right) H(z)$ as soon as $M \geq N$ (more receivers than transmitters). The filter $\left(B_{-}^{\dagger}\left(z^{-1}\right)\right)^{-1}$ is a whitening filter for a process with spectrum $S(z)$. Assuming that $\left(B_{-}^{\dagger}\left(z^{-1}\right)\right)^{-1}$ can be perfectly known, and passing $\underline{x}(z)$ through this filter, we have the following as the output of the whitened matched filter:

$$
\underline{y}(z)=\left(B_{-}^{\dagger}\left(z^{-1}\right)\right)^{-1} \underline{x}(z)=B_{-}(z) \underline{d}(z)+\underline{n}_{1}(z),
$$

where $\underline{n}_{1}(z)$ is a white Gaussian noise with spectrum $N_{0} I, I$ being the $(N \times N)$ identity matrix.

\subsection{WMF implementation using linear prediction}

Several algorithms have been presented in the literature to determine the spectral factors $B_{-}(z)$ and $B_{-}^{\dagger}\left(z^{-1}\right)$ [19]. In the following, based on prediction theory results [9], we briefly explain how to compute an approximation of $\left(B_{-}^{\dagger}\left(z^{-1}\right)\right)^{-1}$.

As we will see, the algorithm based on the prediction theory provides the factorization $S(z)=B_{-}(z) B_{-}^{\dagger}\left(z^{-1}\right)$. Since we want to obtain the spectral factorization given in (5), we begin by factoring $S^{\dagger}(z)=B_{1}(z) B_{1}^{\dagger}\left(z^{-1}\right)$ then we take $B_{-}(z)=B_{1}^{\dagger}(z)$. Denote by $\underline{s}(z)$ a WSS process with spectrum $S^{\dagger}(z)=H^{\dagger}(z) H\left(z^{-1}\right), \underline{i}(z)$ its innovations and $L(z)$ its innovations filter; $\underline{s}(n)$ can be written as

$$
\underline{s}(n)=\sum_{k=0}^{\infty} L(k) \underline{i}(n-k) .
$$

Consider the linear predictor $A(z)=\sum_{k=1}^{\infty} A(k) z^{-k}$ of $\underline{s}(n)$. The estimation $\underline{\hat{s}}(n)$ of $\underline{s}(n)$ in terms of its entire past is then,

$$
\underline{\hat{s}}(n)=\sum_{k=1}^{\infty} A(k) \underline{s}(n-k)=\sum_{k=1}^{\infty} L(k) \underline{i}(n-k) .
$$

It can be approximated by the estimation of $\underline{s}(n)$ in terms of its $D$ most recent past values,

$$
\underline{\hat{s}}(n) \simeq \sum_{k=1}^{D} A(k) \underline{s}(n-k) .
$$

The estimation error is given by

$$
\underline{e}(n)=\underline{s}(n)-\underline{\hat{s}}(n)=L(0) \underline{i}(n) .
$$

Writing the Yule-Walker equations

$$
\begin{aligned}
E\left(\underline{e}(n) \underline{s}(n-k)^{\dagger}\right) & =0, \quad 1 \leq k \leq D, \\
E\left(\underline{e}(n) \underline{s}(n)^{\dagger}\right) & =L(0) L(0)^{\dagger},
\end{aligned}
$$

we obtain

$$
\begin{gathered}
A=(A(1), \ldots, A(D))=R R_{D}^{-1}, \\
R(0)-\sum_{k=1}^{D} A(k) R(k)^{\dagger}=L(0) L(0)^{\dagger} .
\end{gathered}
$$


TABle 1

\begin{tabular}{lccc}
\hline & Channel 1 & Channel 2 & Channel 3 \\
\hline Mean of gain $_{0}$ & 0.540 & 0.369 & 0.159 \\
Standard deviation of gain & 0.114 & 0.107 & 0.121
\end{tabular}

In this expression, $R_{D}$ is the $D N \times D N$ covariance matrix of the random vector $\underline{s}_{D}(n)=\left[\underline{s}(n)^{T}, \ldots, \underline{s}(n-D+1)^{T}\right]^{T}, R(i)=$ $E\left(\underline{s}(k) \underline{s}(k-i)^{\dagger}\right)$, and $R=(R(1), \ldots, R(D))$. From (9), we can write

$$
H^{\dagger}(z) H\left(z^{-1}\right) \simeq A_{1}(z)^{-1} L(0) L(0)^{\dagger}\left(A_{1}^{\dagger}\left(z^{-1}\right)\right)^{-1},
$$

where $A_{1}(z)=\left(I_{N}-\sum_{k=1}^{D} A(k) z^{-k}\right)$.

Hence, by taking the trans-conjugate of this expression, we obtain

$$
H^{\dagger}\left(z^{-1}\right) H(z) \simeq A_{1}\left(z^{-1}\right)^{-1} L(0) L(0)^{\dagger}\left(A_{1}^{\dagger}(z)\right)^{-1} .
$$

Thus, an approximation of the matrix $\left(B_{-}^{\dagger}\left(z^{-1}\right)\right)^{-1}$ is given by $L(0)^{-1} A_{1}\left(z^{-1}\right)$. The implementation of $\left(B_{-}^{\dagger}\left(z^{-1}\right)\right)^{-1}$ is given by solving (11). The autocorrelation matrices $R(i)$ are obtained by identifying the terms in $z^{-i}$ in the equality

$$
H^{\dagger}(z) H\left(z^{-1}\right)=\sum_{i=-\infty}^{+\infty} R(i) z^{-i}
$$

We choose arbitrary $L(0)$ as a lower triangular matrix since we do not care which minimum phase factor is considered.

\subsection{Energy concentration}

Now, we want to verify that the prefiltered MIMO channels have the property of energy concentration as for SISO channels. We start by recalling the property for the SISO case [20].

Theorem 2 (energy concentration [20]). If $B_{-}$is an impulse response of a minimum phase filter, and $H$ a filter having the same spectrum, then for any $n_{0}$,

$$
\sum_{i=0}^{n_{0}}\left|B_{-}(i)\right|^{2} \geq \sum_{i=0}^{n_{0}}|H(i)|^{2} .
$$

It has been shown in [9] that the property of energy concentration stated above holds for minimum phase MIMO channels obtained by spectral factorization for $n_{0}=0$.

Theorem 3 (see [9]). Let $y$ be a WSS stochastic process, let $B_{-}(z)$ be a filter matrix corresponding to the Wold decomposition of $y$ and $W(z)$ a spectral factor of the matrix spectrum of $\underline{y}$ such as $B_{-}(z) B_{-}^{\dagger}\left(z^{-1}\right)=W(z) W^{\dagger}\left(z^{-1}\right)$, then

$$
B_{-}(0) B_{-}(0)^{\dagger} \geq W(0) W(0)^{\dagger} .
$$

In the sequel, we will verify through simulations that this property still holds for orders $n_{0} \geq 1$. In order to measure the
TABle 2

\begin{tabular}{lccc}
\hline & Channel 1 & Channel 2 & Channel 3 \\
\hline Mean of gain $_{1}$ & 0.542 & 0.312 & 0.089 \\
Standard deviation of gain $_{1}$ & 0.138 & 0.118 & 0.081 \\
Mean of gain $_{2}$ & 0.219 & 0.238 & 0.0213 \\
Standard deviation of gain $_{2}$ & 0.094 & 0.104 & 0.0196 \\
\hline
\end{tabular}

gain in terms of energy on the first taps that can be obtained by using the WMF, we consider the following quantities:

$$
\begin{aligned}
\operatorname{gain}_{0}(H) & =\frac{\operatorname{Trace}\left(B_{-}(0)^{\dagger} B_{-}(0)-H(0)^{\dagger} H(0)\right)}{\operatorname{Trace}\left(\sum_{i=0}^{L-1} H(i)^{\dagger} H(i)\right)}, \\
\operatorname{gain}_{n_{0}}(H) & =\frac{\operatorname{Trace}\left(\sum_{i=0}^{n_{0}}\left(B_{-}(i)^{\dagger} B_{-}(i)-H(i)^{\dagger} H(i)\right)\right)}{\operatorname{Trace}\left(\sum_{i=0}^{L-1} H(i)^{\dagger} H(i)\right)} .
\end{aligned}
$$

Table 1 shows the mean and the standard deviation of the measure gain ${ }_{0}$ over 1000 realizations for three types of frequency selective channels described by the standard deviation of the Rayleigh distribution of their taps:

Channel 1: $\underline{\sigma}_{h}=(0.227 ; 0.460 ; 0.688 ; 0.460 ; 0.227)$,

Channel 2: $\underline{\sigma}_{h}=(1 / \sqrt{5} ; 1 / \sqrt{5} ; 1 / \sqrt{5} ; 1 / \sqrt{5} ; 1 / \sqrt{5})$,

Channel 3: $\underline{\sigma}_{h}=(0.716 ; 0.501 ; 0.429 ; 0.214 ; 0.071)$,

where $\underline{\sigma}_{h}=\left(\sigma_{h}(0), \ldots, \sigma_{h}(L-1)\right)$.

These channels were chosen because they have different energy profiles.

Channel 1 has the highest gain since the powers of its delayed paths are larger than that of its direct path. Second in terms of gain, Channel 2 which is quite severe because each path has the same averaged power. Third, Channel 3 which is close to be minimum phase.

Table 2 shows the mean and standard deviation of $\operatorname{gain}_{n_{0}}(H)$ when $n_{0}=1$ and $n_{0}=2$, for the same channels.

For the three channels, Table 2 shows that the energy concentration property is verified when $n_{0}=1$ and $n_{0}=2$. We notice that for Channel 3 the mean and the standard deviation of the different gains are very close. Hence, the gains can be very low for a few realizations. In order to achieve a good tradeoff between complexity and performance, a test can be performed on the calculated gains for each realization of the channel. If $\operatorname{gain}_{n_{0}}(H)$ is less than a determined threshold, the received signals are not prefiltered by the WMF before the equalization, since the improvement will be very little. Otherwise, the WMF is used.

\subsection{Generalized List-type MAP equalizer}

The List-type approach was first proposed for hard-output Viterbi algorithms [6, 7, 8]. A soft version following the idea of the MAP algorithm has been proposed by Penther et al. [5]. We present here a generalization of this 
soft-input/soft-output algorithm to MIMO channels. As explained in $[6,7,8]$, the trellis has $Q^{(J-1) N}$ states where $J$ is the reduced memory of the channel $(J<L)$ and $Q$ is the constellation size. The first $J$ taps are processed by the trellis transitions of the List-type equalizer and the remaining taps are processed by a per-survivor processing with $S$ survivors at each state.

Our objective is to compute the a posteriori probabilities (APP) $P\left(d_{i}(n) \mid y\right)$ for $1 \leq i \leq N$ and $0 \leq n \leq T-1$, given the received vector, after matched filtering and whitening, during a burst $\underline{y}=\left(\underline{y}(0)^{T}, \ldots, \underline{y}(T-1)^{T}\right)^{T}$.

$\mu(n)=\left(\underline{d}^{T}(n-1), \underline{d}^{T}(n-2), \ldots, \underline{d}^{T}(n-J+2), \underline{d}^{T}(n-J+1)\right)$

be the state at time $n$. As described in $[3,4]$, the APP are obtained after calculation of the forward probability $\alpha_{n}(\mu)$ and the backward probability $\beta_{n}(\mu)$. Here, the forward probability $\alpha_{n}^{S_{i}}(\mu)$ will also depend on the survivors $S_{i}(i=1, \ldots, S)$. At time $n$, a state $\mu$ is associated to a survivor list $S_{i, n}$ and a forward probability list $\alpha_{n}^{S_{i}}(\mu)$. At time $n+1, S Q^{N}$ branches converge to the following state $\mu^{\prime}$ and their path metrics are defined as $\alpha_{n}^{S_{i}}(\mu) \gamma_{n}^{S_{i}}\left(\mu, \mu^{\prime}\right)$ where $\gamma_{n}^{S_{i}}\left(\mu, \mu^{\prime}\right)$ is the branch transition probability between states $\mu$ and $\mu^{\prime}$ for the survivor $S_{i}$. This transition can be expressed as the product of an a priori probability and the channel transition probability

$$
\gamma_{n}^{S_{i}}\left(\mu, \mu^{\prime}\right)=p\left(\underline{y}(n) \mid \mu, \mu^{\prime}\right) P\left(\mu^{\prime} \mid \mu\right) .
$$

Since the WMF keeps the noise white and Gaussian, the channel transition probability is given by,

$$
\begin{aligned}
& p\left(\underline{y}(n) \mid \mu, \mu^{\prime}\right)=\frac{1}{\left(\pi \sigma^{2}\right)^{N}} \\
& \times \exp \left(-\frac{\left|\underline{y}(n)-\sum_{l=0}^{J-1} B_{-}(l) \underline{\tilde{d}}(n-l)-\sum_{l=J}^{L-1} B_{-}(l) \underline{\hat{d}}(n-l)\right|^{2}}{\sigma^{2}}\right),
\end{aligned}
$$

where $\underline{\tilde{d}}(n-l)=\left(\tilde{d}_{1}(n-l), \ldots, \tilde{d}_{N}(n-l)\right)$ is the vector of elements of the trellis transition and $\hat{d}(n-l)=\left(\hat{d}_{1}(n-\right.$ $\left.l), \ldots, \hat{d}_{N}(n-l)\right)$ is the vector of the symbols estimated during the per-survivor processing for a given survivor among $S$. All symbols are assumed equally likely, so $P\left(\mu^{\prime} \mid \mu\right)=1 / Q^{N}$.

The $S Q^{N}$ path metrics are sorted by order and the $S$ highest will define, for the state $\mu^{\prime}$ at time $n+1$, the survivor list $S_{i^{\prime}, n+1}$ and the forward probabilities $\alpha_{n+1}^{S_{i^{\prime}}}\left(\mu^{\prime}\right)$. Each forward probability $\alpha_{n+1}^{S_{i}}\left(\mu^{\prime}\right)$ is calculated as the sum of all the path metrics leading to $\mu^{\prime}$ which are inferior or equal to the path metric associated to $S_{i^{\prime}}$ [5]. The backward probability $\beta_{n}(\mu)$ is calculated recursively using the survivors obtained in the preceding step as

$$
\beta_{n}(\mu)=\sum_{\mu^{\prime}} \sum_{i=1}^{S} \gamma_{n}^{S_{i}}\left(\mu, \mu^{\prime}\right) \beta_{n+1}\left(\mu^{\prime}\right) .
$$

Let $\left\{m_{1}, \ldots, m_{Q}\right\}$ be the constellation points. The equalizer calculates the APP $P\left(d_{i}(n)=m_{q} \mid \underline{y}\right)$ for each possible symbol $d_{i}(n)=m_{q}, q=1, \ldots, Q$, as

$$
\begin{aligned}
P( & \left.d_{i}(n)=m_{q} \mid \underline{y}\right) \\
= & \sum_{f=1}^{Q^{N-1}} \sum_{\mu=1}^{(U-1) N} \sum_{j=1}^{S} \alpha_{n}^{S_{j}}(\mu) \gamma_{n}^{S_{j}}\left(\mu, S_{f}^{m_{q}}(\mu)\right) \beta_{n+1}\left(S_{f}^{m_{q}}(\mu)\right),
\end{aligned}
$$

where $S_{f}^{m_{q}}(\mu)$, for $1 \leq f \leq Q^{N-1}$, are the states following the state $\mu$ if the input symbol corresponding to the $i$ th transmitting antenna is $m_{q}$.

The probability that a coded bit $c_{i}(k)$ is equal to 0 is then calculated as

$$
P\left(c_{i}(k)=0 \mid \underline{y}\right)=\sum_{d_{i}(n) \in d^{0}} P\left(d_{i}(n) \mid \underline{y}\right),
$$

where $n=\left\lfloor k / \log _{2}(Q)\right\rfloor, d^{0}$ is the set of symbols $d_{i}(n)$ corresponding to $c_{i}(k)=0$.

The probability $P\left(c_{i}(k)=1 \mid \underline{y}\right)$ is obtained as

$$
P\left(c_{i}(k)=1 \mid \underline{y}\right)=1-P\left(c_{i}(k)=0 \mid \underline{y}\right) .
$$

Our equalizer encompasses the MAP algorithm obtained by letting $J=L$ and $S=1$ and the soft DDFSE obtained by letting $J<L$ and $S=1$.

\subsection{Simulation results}

Since we have been interested so far in the equalization part, we consider the performance of the receiver for a simplified system without encoding and interleaving. The receiver is composed of the WMF and the soft List-type MAP equalizer. The equalizer uses the soft-input/soft-output algorithm presented in Section 3.4.

Figures 2 and 3 show the performance of the List-type equalizer for the three channels defined previously with and without the WMF. The different reduced memory sizes are $J=2$ and $J=3$. The modulation is the BPSK. The number of survivors is $S=2$.

We notice that the use of the WMF yields a significant gain in performance for the three channels. As expected from Section 3.1, the channel gaining most improvement by the use of the WMF is Channel 1 followed by Channel 2 then Channel 3. As shown in Figure 3, the improvement is less important when the reduced memory $J$ of the channel is higher.

\section{LOW-COMPLEXITY TURBO DETECTOR}

The previous improvement is however not sufficient to compensate the loss with respect to the single user bound. Next, we propose to use a turbo detector in order to take into account the coded structure of transmitted sequences. It is composed of the generalized List-type MAP equalizer and a SISO decoder using the BCJR-MAP algorithm proposed by Bahl et al. [3]. 


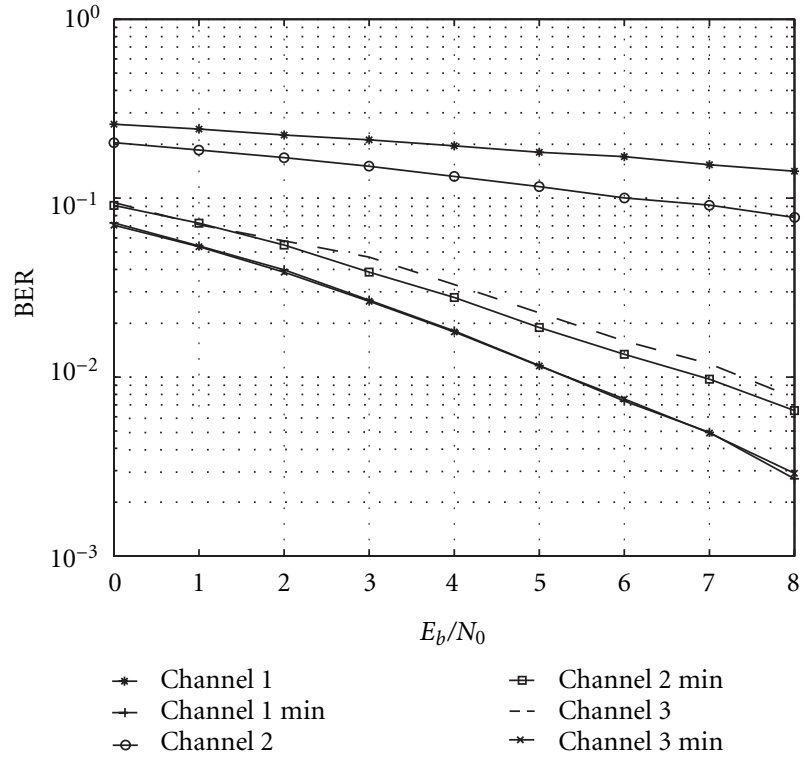

FIgUre 2: Performance of the List-type MAP equalizer with and without the WMF for a channel reduced memory $J=2$.

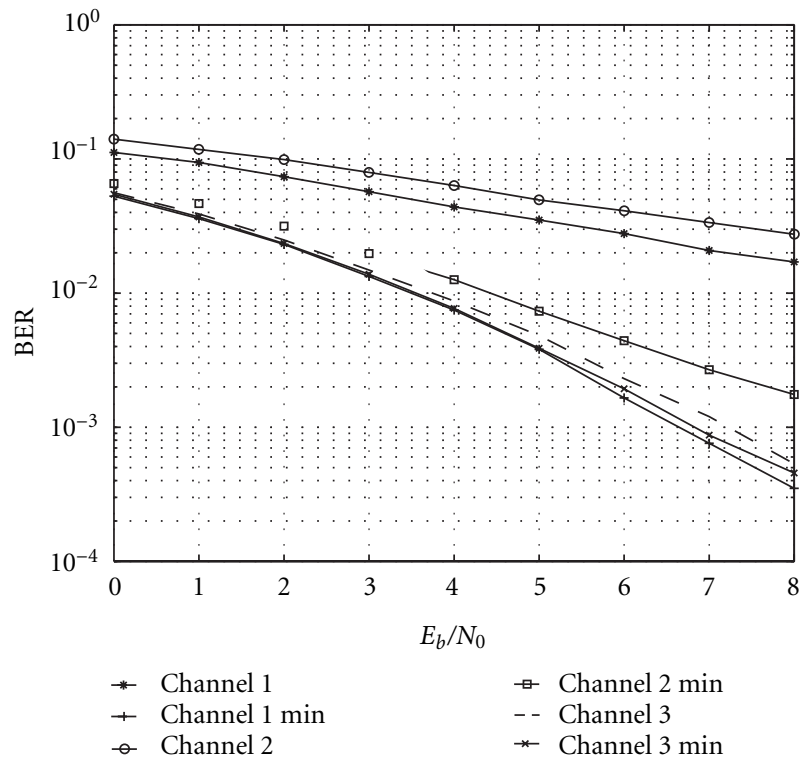

FIgURe 3: Performance of the List-type MAP equalizer with and without the WMF for a channel reduced memory $J=3$.

\subsection{Principle of turbo detection}

Figure 4 shows the iterative receiver system. On the first iteration, the List-type MAP equalizer generates the a posteriori probabilities $P_{\mathrm{eq}}\left(c_{i}(k) \mid y\right)$ for $1 \leq i \leq N$, given the whole received vector during a burst. These probabilities are deinterleaved and multiplexed. The decoder uses these information to calculate the APP for the information bits and for the coded bits using the MAP algorithm [3].

Extrinsic information are computed from a posteriori coded bits probabilities $P_{\operatorname{dec}}\left(c_{i}(n) \mid \underline{y}\right)$ as

$$
P_{\mathrm{dec}}^{\mathrm{ext}}\left(c_{i}(n) \mid \underline{y}\right)=\frac{P_{\mathrm{dec}}\left(c_{i}(n) \mid \underline{y}\right)}{P_{\mathrm{eq}}\left(c_{i}(n) \mid \underline{y}\right)} .
$$

These information are provided to the equalizer as a priori information on the next iteration in order to obtain more reliable soft outputs. The equalizer computes the APP $P_{\mathrm{eq}}\left(c_{i}(k) \mid y\right)$ on the coded bits and provides the extrinsic information $P_{\mathrm{eq}}^{\mathrm{ext}}\left(c_{i}(k) \mid y\right)$ to the decoder. The probabilities $P_{\mathrm{eq}}^{\mathrm{ext}}\left(c_{i}(k) \mid \underline{y}\right)$ are calculated as

$$
P_{\mathrm{eq}}^{\mathrm{ext}}\left(c_{i}(k) \mid \underline{y}\right)=\frac{P_{\mathrm{eq}}\left(c_{i}(k) \mid \underline{y}\right)}{P_{\operatorname{dec}}^{\operatorname{ext}}\left(c_{i}(k) \mid \underline{y}\right)} .
$$

After some iterations, hard decisions on the input information bits are taken by the decoder.

It is important that the receiver modules exchange extrinsic information in order to minimize the correlation between the a priori information used by a module and its previous decisions. To fully realize the potential of the iterative receiver, the streams of coded symbols are fed to independent random interleavers before they are transmitted. Interleavers help to break the correlation of the encoder output. They also guarantee that the a priori information provided to the equalizer are almost independent. The use of different interleavers may improve the performance of the iterative receiver.

\subsection{Equalizer for the iterative processing}

For the first iteration, the equalizer is the List-type MAP algorithm described in Section 3. For the next iterations, the MAP decoder provides the equalizer with extrinsic information and only the calculation of the transition probabilities $\gamma_{n}^{S_{i}}\left(\mu, \mu^{\prime}\right)$ is changed. More precisely, the a priori probabilities in (20) are now calculated as,

$$
P\left(\mu^{\prime} \mid \mu\right)=\prod_{i=1}^{N} P_{\mathrm{dec}}^{\mathrm{ext}}\left(c_{i} \mid \underline{y}\right),
$$

where $c_{i}, i=1, \ldots, N$ correspond to the transition $\left(\mu, \mu^{\prime}\right)$ and $P_{\mathrm{dec}}^{\mathrm{ext}}\left(c_{i} \mid \underline{y}\right)$ comes from (26).

\subsection{Simulation results}

In the following simulations, the calculation of the WMF is performed by using a predictor of degree $D=10$. We focus on Channel 2 since it is a severe channel, so it is a good test for evaluating the performance of our receiver.

Figure 5 shows the performance for one to three iterations of the turbo receiver with and without the WMF. When the WMF is not used the number of survivors $S$ is set to 4 and when it is used the number of survivors is set to 1 and 2. We notice that for the coded system the use of the WMF yields a significant improvement even if the number of survivors is low $(S=1$ and $S=2)$. We can conclude that the gain achieved when prefiltering the received signal with a WMF 


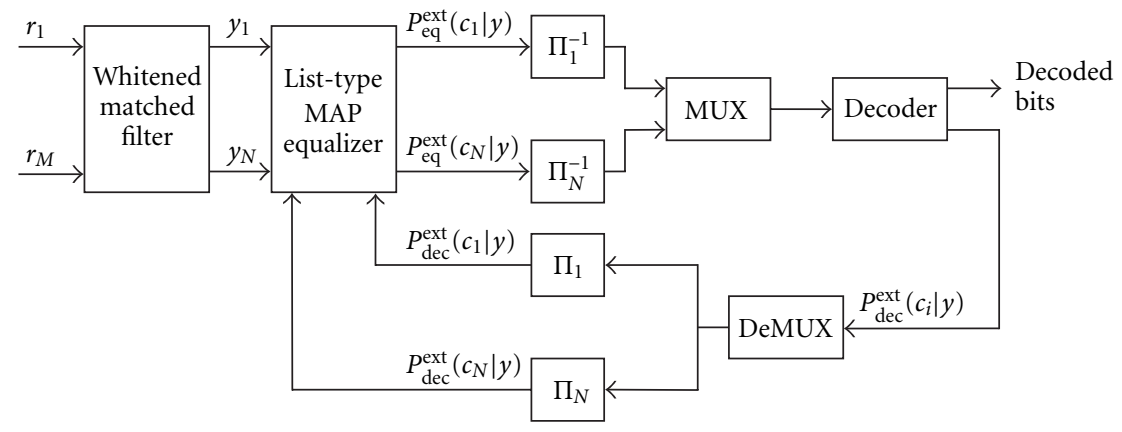

FIGURE 4: Low-complexity turbo detector.

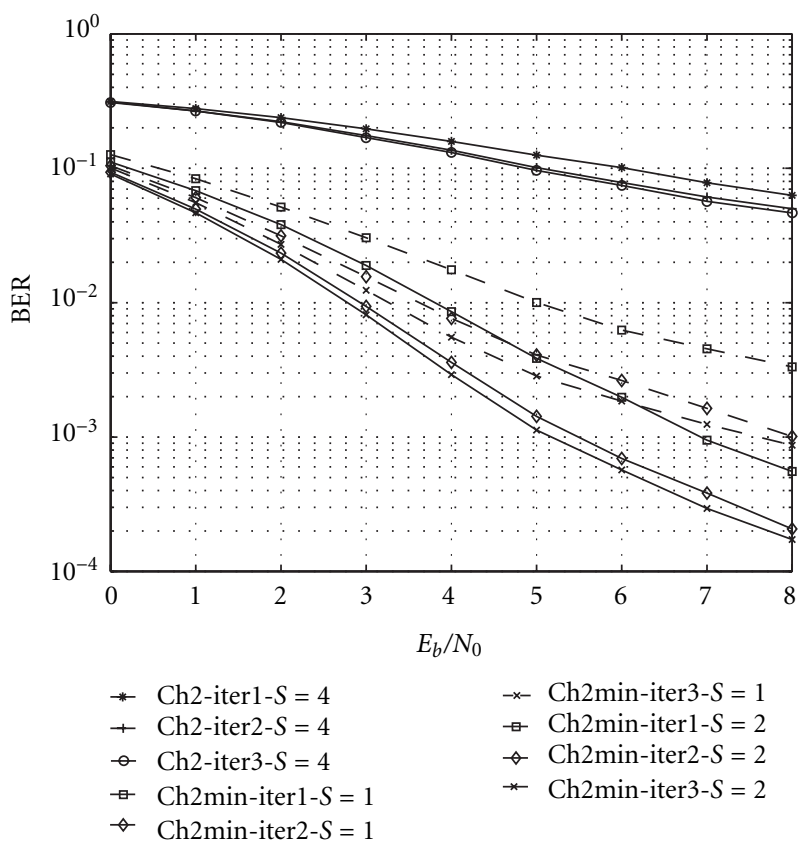

FIGURE 5: BER performance of the iterative detector $(J=2)$ on Channel 2 versus $E_{b} / N_{0}$ : Ch2-iter corresponds to a receiver with a 4 survivor detector and $\mathrm{Ch} 2 \mathrm{~min}$-iter corresponds to the receiver with the WMF and a dfse ( $S=1$, dotted lines) or a 2-survivor detector $(S=2)$.

is dramatically more important than that obtained when increasing the number of survivors. The gain achieved via the iterative processing is also obvious. We notice that most of the improvement is achieved in the second iteration. For a $\mathrm{BER}=10^{-3}$, the iterative processing yields an improvement of $1.8 \mathrm{~dB}$, at the third iteration when the WMF is used with the 2-survivor detector.

\section{CONCLUSION}

In this paper, we have proposed a low-complexity turbo detector for space-time coded frequency selective channels. Our detector is a suboptimal variant of the MAP algorithm based on state-reduction and per-survivor processing. In order to compensate the loss due to state-reduction, we have designed a multidimensional whitened matched filter used as a prefilter for the turbo detector. Simulation results show that this prefiltering improves significantly the receiver performance particularly over severe channels. Our receiver achieves a good tradeoff between complexity and performance. In fact, we have decreased exponentially the complexity of the optimum MAP detector. Moreover, thanks to the prefiltering and the iterative process, the performance has been dramatically improved. Thus, our turbo detector is a good candidate for multi-antenna systems mainly when the memory of the channel is large and the constellation used has a high order.

\section{REFERENCES}

[1] G. J. Foschini and M. J. Gans, "On limits of wireless communications in a fading environment when using multiple antennas," Wireless Personal Communications, vol. 6, no. 3, pp. 311-335, 1998.

[2] G. K. Kaleh, "Channel equalization for block transmission systems," IEEE Journal on Selected Areas in Communications, vol. 13, no. 1, pp. 110-121, 1995.

[3] L. R. Bahl, J. Cocke, F. Jelinek, and J. Raviv, "Optimal decoding of linear codes for minimizing symbol error rate," IEEE Transactions on Information Theory, vol. 20, no. 2, pp. 284$287,1974$.

[4] G. Bauch, A. F. Naguib, and N. Seshadri, "MAP equalization of space-time coded signals over frequency selective channels," in Wireless Communications and Networking Conference, New Orleans, La, USA, September 1999.

[5] B. Penther, D. Castelain, and H. Kubo, "A modified turbodetector for long delay spread channels," in International Symposium on Turbo Codes, Brest, France, September 2000.

[6] A. Duel-Hallen and C. Heegard, "Delayed decision-feedback sequence estimation," IEEE Trans. Communications, vol. 37, no. 5, pp. 428-436, 1989.

[7] T. Hashimoto, "A list-type reduced-constraint generalization of the Viterbi algorithm," IEEE Transactions on Information Theory, vol. 33, no. 6, pp. 866-876, 1987.

[8] H. Kubo, K. Murakami, and T. Fujino, "A list-output Viterbi equalizer with two kinds of metric criteria," in Proc. IEEE International Conf. on Universal Pers. Commun., pp. 1209-1213, October 1998.

[9] P. E. Caines, Linear Stochastic Systems, Wiley, New York, USA, 1988.

[10] A. Duel-Hallen, "Equalizers for multiple input/multiple output channels and PAM systems with cyclostationary input sequences," IEEE Journal on Selected Areas in Communications, vol. 10, no. 3, pp. 630-639, 1992. 
[11] A. Duel-Hallen, "A family of multiuser decision-feedback detectors for asynchronous code-division multiple-access channels," IEEE Trans. Communications, vol. 43, no. 2-4, pp. 421434, 1995.

[12] G. Caire, G. Taricco, and E. Biglieri, "Bit-interleaved coded modulation," IEEE Transactions on Information Theory, vol. 44, no. 3, pp. 927-946, 1998.

[13] E. Zehavi, "8-PSK trellis codes for a Rayleigh channel," IEEE Trans. Communications, vol. 40, no. 5, pp. 873-884, 1992.

[14] C. Berrou, A. Glavieux, and P. Thitimajshima, "Near Shannon limit error-correcting coding and decoding: turbo codes," in Proc. IEEE International Conf. on Communications, pp. 10641070, Geneva, Switzerland, May 1993.

[15] X. Wang and H. V. Poor, "Iterative (turbo) soft interference cancellation and decoding for coded CDMA," IEEE Trans. Communications, vol. 47, no. 7, pp. 1046-1061, 1999.

[16] A. Roumy, I. Fijalkow, and D. Pirez, "Turbo multiuser detection for coded asynchronous DS-CDMA over frequency selective channels," J. on Commun. Networks, vol. 3, no. 3, pp. 202-210, 2001, special issue on multiuser detector.

[17] G. Bauch and N. Al-Dhahir, "Iterative equalization and decoding with channel shortening filters for space-time coded modulation," in Proc. IEEE Vehicular Technology Conference, pp. 1575-1582, Boston, Mass, USA, September 2000.

[18] N. Al-Dhahir, "FIR channel-shortening equalizers for MIMO ISI channels," IEEE Trans. Communications, vol. 49, no. 2, pp. 213-218, 2001.

[19] V. Kucera, "Factorization of rational spectral matrices: a survey of methods," in Proc. IEE International Conference on Control, vol. 2, pp. 1074-1078, Edinburgh, 1991.

[20] A. Papoulis, Signal Analysis, McGraw-Hill, New York, NY, USA, 1977.

Noura Sellami was born in Tunisia, in 1975. She received the preliminary doctorate certificate in signal processing from University of Cergy-Pontoise, France, and the engineer diploma from École Nationale Supérieure de l'Électronique et de ses Applications (ENSEA), France, in 1999. She is currently working toward the Ph.D. degree at University of Cergy-Pontoise, France.

Inbar Fijalkow received the Engineering M.S. and Ph.D. degrees from École Nationale Supérieure de Télécommunications (ENST), Paris, France, in 1990 and 1993, respectively. In 1993-1994, she was a Research Associate at Cornell University, NY, USA. During 1994-1999, she was an Associate Professor, since 1999 she has been a Professor. She is member of the Laboratory ETIS (ENSEA-University of Cergy-

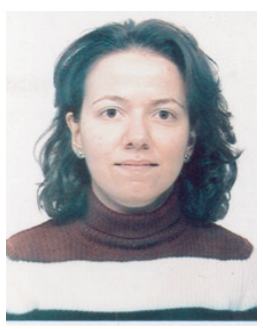
Pontoise), France. In 1998, she was a visiting researcher at the Australian National University (ANU), Canberra, Australia. Her current research interests are in signal processing applied to digital communications: adaptive and iterative (turbo) processing, blind deconvolution/equalization of multiple sources and sensors systems. She is member of the board of the GDR ISIS, which is the CNRS research group on signal image and vision processing. She has been associate editor of the IEEE Transactions on Signal Processing since August 2000.
Mohamed Siala was born in 1965 in Tunisia. He received the "Diplôme d'Ingénieur" from "École Polytechnique, Palaiseau, France," in 1988, the "Diplôme d'Ingénieur" from "École $\mathrm{Na}$ tionale Supérieure des Télécommunications (ENST)," Paris, France, in 1990 and the "Doctorat de l'ENST," Paris, France, in 1995. From 1990 to 1992 , he was with Alcatel Radio-Telephones, Colombes,

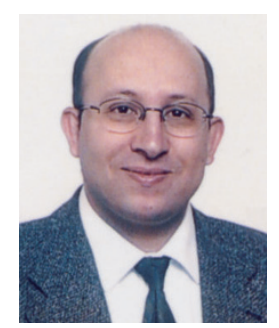
France, working on the GSM physical layer. In 1995, he joined Wavecom, Issy-les-Moulineaux, France, where he worked on IOTA multicarrier modulations for terrestrial digital TV and channel estimation for the ICO project. From 1997 to 2001, he worked at France Télécom R\&D, Issy-les-Moulineaux, France, on the physical layer of the UMTS system. In 2001, he joined "École Supérieure des Communications de Tunis (SUPCOM)," Tunis, Tunisia, where he is an Associate Professor. His research interests are in the area of digital communications with special emphasis on channel estimation, modulation and coding for mobile communications. 